\title{
MICRO-SCALE TECHNOLOGIES FOR CELL MECHANICS: EXPERIMENTAL AND MODELLING APPROACHES
}

\author{
FEDERICA CASELLI, NICOLA A. NODARGI, PAOLO BISEGNA (*)
}

SUNTo. - La meccanica cellulare, disciplina ponte fra biologia cellulare e meccanica, può grandemente giovarsi delle recenti tecnologie di microscala, che, grazie alla loro efficacia in termini di capacità di processo, costi e facilità di fabbricazione, rendono possibili nuove direzioni di ricerca. In questo lavoro sono discussi due esempi di tali tecnologie: la citometria microfluidica ad impedenza e l'elettrodeformazione degli eritrociti. La prima è una tecnologia lab-on-chip che offre una soluzione semplice e non invasiva per identificare e monitorare le caratteristiche biofisiche e meccaniche a livello di singola cella, senza richiedere alcuna marcatura. La seconda, complementare alla prima, rende possibile la deformazione cellulare tramite un campo elettrico applicato.

$$
* * *
$$

Abstract. - Cell mechanics is a discipline that bridges cell biology with mechanics. Emerging microscale technologies are opening new venues in the field, due to their costeffectiveness, relatively easy fabrication, and high throughput. Two examples of those technologies are discussed here: microfluidic impedance cytometry and erythrocyte electrodeformation. The former is a lab-on-chip technology offering a simple, non-invasive, label-free method for counting, identifying and monitoring cellular biophysical and mechanical function at the single-cell level. The latter is a useful complement to the former, enabling cell deformation under the influence of an applied electric field.

\section{INTRODUCTION}

Robert Hooke discovered the law of elasticity which bears his name in 1660. He first described this discovery in the anagram "ceiiinosssttuv",

This work was supported by the Scientific Independence of Young Researchers Programme (SIR 2014) under Grant RBSI14TX20-MUSIC "Multidimensional Single-Cell Microfluidic Impedance Cytometry".

(*) Department of Civil Engineering and Computer Science, University of Rome Tor Vergata, 00133 Rome, Italy. Email: bisegna@uniroma2.it 
whose solution he published in 1678 as "Ut tensio, sic vis", stating the linear variation of tension with extension in an elastic spring. Hooke published his book Micrographia in 1665, where he coined the term "cell" for describing biological organisms, as suggested by the resemblance of cork cells to cells of a honeycomb. Despite being studied by the same eminent scientist in the seventeenth century, the field of elasticity (more generally, mechanics) and the study of biological cells remained separated for centuries.

The mechanical properties of cells have recently been shown to be useful markers of cell state. In particular, there is growing evidence that cell deformability may provide a label-free biomarker for determining e.g. metastatic potential, degree of differentiation, or cell activation [13].

The study of cell mechanics is a modern multidisciplinary field that bridges cell biology with mechanics, focusing on the mechanical properties of living cells and how they relate to cell function. Since a typical cell body is about $10 \mu \mathrm{m}$ in diameter, to capture a complete picture of mechanical interactions and physical properties of cells, the size or resolution of the tools utilized in cellular biophysical studies has to be in that order of size or smaller [36]. With recent advances in microtechnology and microfabrication methods, the field of biological micro-electromechanical-systems has been opened [36]. These devices are helping to elucidate the mechanical properties of cells, the nature of cellular forces, and responses that cells have to external forces. Information gained from these studies is utilized in computational models that address cell mechanics as a collection of biomechanical and biochemical processes. These models are advantageous in explaining experimental observations by providing a framework of underlying cellular mechanisms. They also enable predictive, in silico studies, which would otherwise be difficult or impossible to perform with current experimental approaches [36].

Several techniques are currently available to study cell mechanics. As an example, methods based on atomic force microscopy [11] usually exert local forces on a cell. Therefore, they suffer from low throughput and direct contact with the cell surface may cause active cellular responses. Single-cell-based techniques such as micropipette aspiration [40] have modest throughput, but they also cannot avoid cell-tool interactions either. Optical techniques such as optical tweezers [26] minimize active cell responses when deforming cells. The laser induced forces can be precisely controlled by output power.

Microfluidic techniques provide versatility in cell mechanics under 
precisely controlled fluid flow [36]. In this work two specific examples are discussed: microfluidic impedance cytometry and erythrocyte electrodeformation. The former is a lab-on-chip technology offering a simple, noninvasive, label-free method for counting, identifying and monitoring cellular biophysical and mechanical function at the single-cell level. The latter is a useful complement to the former, enabling cell deformation under the influence of an applied electric field.

\section{MiCROFLUIDIC IMPEDANCE CYTOMETRY}

Microfluidic impedance cytometry is a non-invasive technique for the electrical characterization of single particles and cells $[10,35]$. Compared with traditional approaches like flow cytometry, its main advantages are the label-free nature, requiring minimal sample processing, and the potential for low-cost and portable implementation. The basic principle is as follows: i) an AC electric field is established in a microchannel equipped with microelectrodes and filled with a conductive fluid; ii) the field perturbation induced by the passage of a flowing particle is measured as an electric differential current signal.

The measured signal is processed to extract valuable information for particle characterization. As an example, information on size, membrane capacitance, and cytoplasm conductivity of biological cells can be obtained depending on the frequency of the AC electric field (e.g., [22]).

\subsection{Operating principle}

The conventional operation mode of the standard impedance chip comprising two pairs of facing electrodes is depicted in Fig. 1(a) -(b): an AC voltage is applied to the top stimulating electrodes, and the differential current flowing through the bottom measuring electrodes is collected. The passage of a particle is detected as two opposite pulses with the same amplitude [32,5], respectively recorded when the particle is between the left or right pair of electrodes (Fig. 1(c), simulation results [6,9]). Pulse amplitude primarily depends on the change in the transversal current (i.e., current flowing through a measuring electrode originating from the opposite stimulating one) upon the passage of a particle. Pulse amplitude at low frequency is therefore a measure of particle volume $[22,35]$. However, the electric field within the sensing region is non-uniform due to fringing effects [34]. Hence, pulse amplitude also depends on particle 
(a)

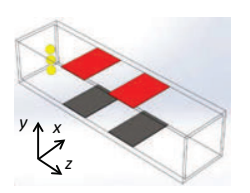

(b)

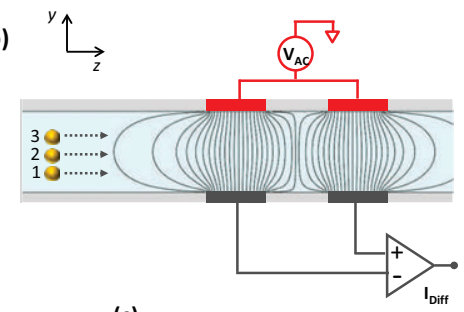

(c)

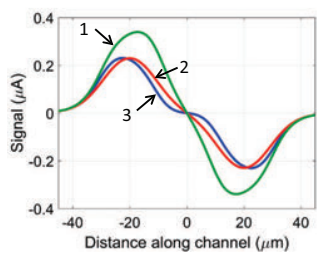

(d)

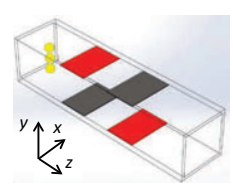

(e)

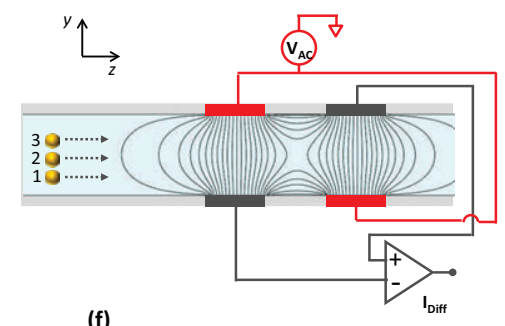

(f)

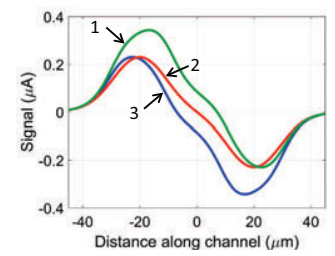

Fig. 1. (a)-(c) Conventional operation mode. (a) 3D view and (b) side view cartoon. An AC voltage is applied to the top stimulating electrodes and the differential current flowing through the bottom measuring electrodes is collected. Typical current lines are shown. (c) Differential signals (real part, simulation results) produced by an insulating particle passing through the sensing region along three different trajectories: close to the bottom of the channel (curve 1), through the middle of the channel (curve 2) and close to the top of the channel (curve 3). Each curve consists of a pair of opposite Gaussian pulses with the same amplitude. (d)-(f) Proposed operation mode. (d) $3 D$ view and (e) side view cartoon. An AC voltage is applied to diagonally opposite stimulating electrodes and the differential current flowing through the remaining measuring electrodes is collected. (f) Differential signals relevant to the three different trajectories considered in panel (c) (real part, simulation results). Each curve consists of a pair of opposite Gaussian pulses with different amplitude. Pulse amplitude difference depends on particle trajectory beight.

trajectory height, i.e. y-coordinate of particle center (Fig. 1(c)). A further contribution to pulse amplitude comes from the cross current between the left and right pair of electrodes (i.e., current flowing through 


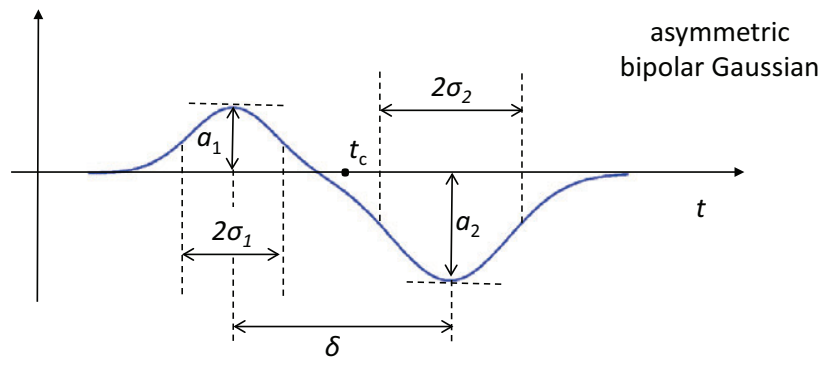

Fig. 2. Asymmetric bipolar Gaussian template used to fit the experimental traces collected using the proposed wiring scheme.

a measuring electrode originating from the diagonally opposite stimulating one). As a consequence of the cross current, pulse amplitude is higher when the particle flows closer to the measuring electrodes than to the stimulating ones (the system is asymmetric top to bottom) [32]. This results in a positional dependence of the measured signals, i.e. identical particles flowing along different trajectories in the microchannel provide different signals $[32,18]$, thus produceing blurring of estimated particle properties.

Since the equations governing the electric conduction in the cytometer are elliptic, an analogy can be established with the deformation of an elastic body. Electrodes are analogous to restrained portions of the boundary, and applied voltage is analogous to applied displacement. Accordingly, the measured signal is analogous to the influence line of the difference in the constraint forces, generated by a "mobile" inclusion traveling through the body. On the basis of this analogy, signal positional dependence is certainly expected.

A new operation mode [7] is depicted in Fig. 1(d)-(e): an AC voltage is applied to diagonally opposite stimulating electrodes and the differential current flowing through the remaining measuring electrodes is collected. This simple modification is suggested by the following heuristic observation. A particle flowing in the lower half of the channel (Fig. 1(e), trajectory 1) is located closer to the bottom measuring electrode than to the top stimulating one when detected by the left pair of electrodes. Then, it is located farther to the top measuring electrode than to the bottom stimulating one when detected by the right pair of electrodes. Accordingly, the left pulse of the recorded signal is expected to have higher 
amplitude than the right pulse, as confirmed by numerical simulations (Fig. 1(f), curve 1). The opposite behaviour is expected for particles flowing in the upper half of the channel (Fig. 1(f), curve 3), whereas particles traveling through the middle of the channel yield pulses with equal amplitude (Fig. 1(f), curve 2). This suggests that pulse amplitude difference is related to particle trajectory height, thus lending itself as a metric useful to treat the positional dependence issue (cf. also the simulation results reported in [8]). On the other hand, pulse amplitude average is a measure of particle volume (at low frequency), and its cube root is proportional to particle diameter. The relative difference of pulse amplitudes (i.e., pulse amplitude difference divided by pulse amplitude average) is here chosen, in order to obtain a metric independent of particle size.

An asymmetric bipolar Gaussian template (Fig. 2) can be conveniently fitted to signal traces (Fig. I(f)) in order to extract the left- and right-pulse amplitudes. The template is obtained as the difference of two shifted Gaussian pulses with different amplitude and width, as follows:

$$
s(t)=g_{1}\left(t-t_{c}+\delta / 2\right)-g_{2}\left(t-t_{c}-\delta / 2\right),
$$

with:

$$
g_{1}(t)=a_{1} e^{-t^{2} /\left(2 \sigma_{1}^{2}\right)}, \quad g_{2}(t)=a_{2} e^{-t^{2} /\left(2 \sigma_{2}^{2}\right)} .
$$

It depends on the following parameters: central time moment, $t_{c}$; transit time, $\delta$; pulse width controls, $\sigma_{1}$ and $\sigma_{2}$; pulse amplitude controls, $a_{1}$ and $a_{2}$.

According to the previous discussion, an estimate $D$ of the particle diameter $d$ is supplied by the cube root of the mean value of the pulse amplitude controls, $\left(a_{1}+a_{2}\right) / 2$ :

$$
D=G\left[\left(a_{1}+a_{2}\right) / 2\right]^{1 / 3},
$$

where $G$ is a gain factor to account for the electronic circuitry. The estimate $D$ is referred to as "electrical" diameter. In addition, the pulse amplitude relative difference, $\Delta$ is introduced:

$$
\Delta=\frac{a_{2}-a_{1}}{\left(a_{1}+a_{2}\right) / 2} .
$$

This new metric enables a simple compensation procedure of the signal impedance for off-centre particles, thus solving the positional dependence issue. 


\subsection{Experimental}

As typical facing electrode chip (Fig. I), a commercial microfluidic chip for electrical impedance spectroscopy of single cells was used (EIS chip, Micronit). The chip has a straight through channel $28 \mu \mathrm{m}$ deep and $30 \mu \mathrm{m}$ wide. There are two sets of double electrodes $(\mathrm{Pt})$. Electrode dimension along channel axis is $20 \mu \mathrm{m}$, with planar separation of $20 \mu \mathrm{m}$ and $28 \mu \mathrm{m}$ depth separation as based on channel depth.

The proposed method is validated by means of polystyrene beads (Sigma-Aldrich and Polysciences). Beads with diameters of 5.2, 6, and $7 \mu \mathrm{m}$ were suspended in PBS (Phosphate-Buffered Saline) with $0.1 \%$ Tween 20 to avoid bead aggregation, and sufficient sucrose to match the density of the beads. Sample concentration and conductivity were approximately 1000 beads $/ \mu \mathrm{l}$ and $1.1 \mathrm{~S} / \mathrm{m}$, respectively.

A syringe pump (Harvard Apparatus) operating at $10 \mu \mathrm{l} / \mathrm{min}$ was used for fluidic control. Electrical measurements were performed using an impedance spectroscope (HF2IS, Zurich Instruments), along with a transimpedance amplifier (HF2TA, Zurich Instruments) for signal conditioning. A signal of $4 \mathrm{~V}$ at $0.5 \mathrm{MHz}$ was applied to the stimulating electrodes and the differential current flowing through the virtualground measuring electrodes was demodulated and recorded (readout rate $115 \mathrm{k}$ sample per second, $20 \mathrm{kHz}$ filter bandwidth).

\subsection{Results}

Fig. 3 shows a density plot for $6 \mu \mathrm{m}$ diameter beads, with the pulse amplitude relative difference $\Delta$ (equation (4)) plotted against the electrical diameter $D$ (equation (3)), as measured using the proposed wiring scheme. Examples of experimental single particle signals are shown in panels (i)-(vii), along with arrows indicating the position of the particle on the density plot. The difference in the amplitudes of the two opposite pulses predicted in simulation (Fig.1(f)) is experimentally confirmed. In addition to the experimental signals, their fitting templates are also shown in panels (i)-(vii), showing excellent accuracy.

Fig. 4 (a) to (c) shows a density plot of the pulse amplitude relative difference $\Delta$ vs the electrical diameter $D$ for three different bead sizes (measured separately using the proposed wiring scheme, and collected together in Fig. 4(d)). In each case the same parabolic trend is observed. This data can be fitted to a quadratic function:

$$
D=a\left[1+b(\Delta-c)^{2}\right]
$$



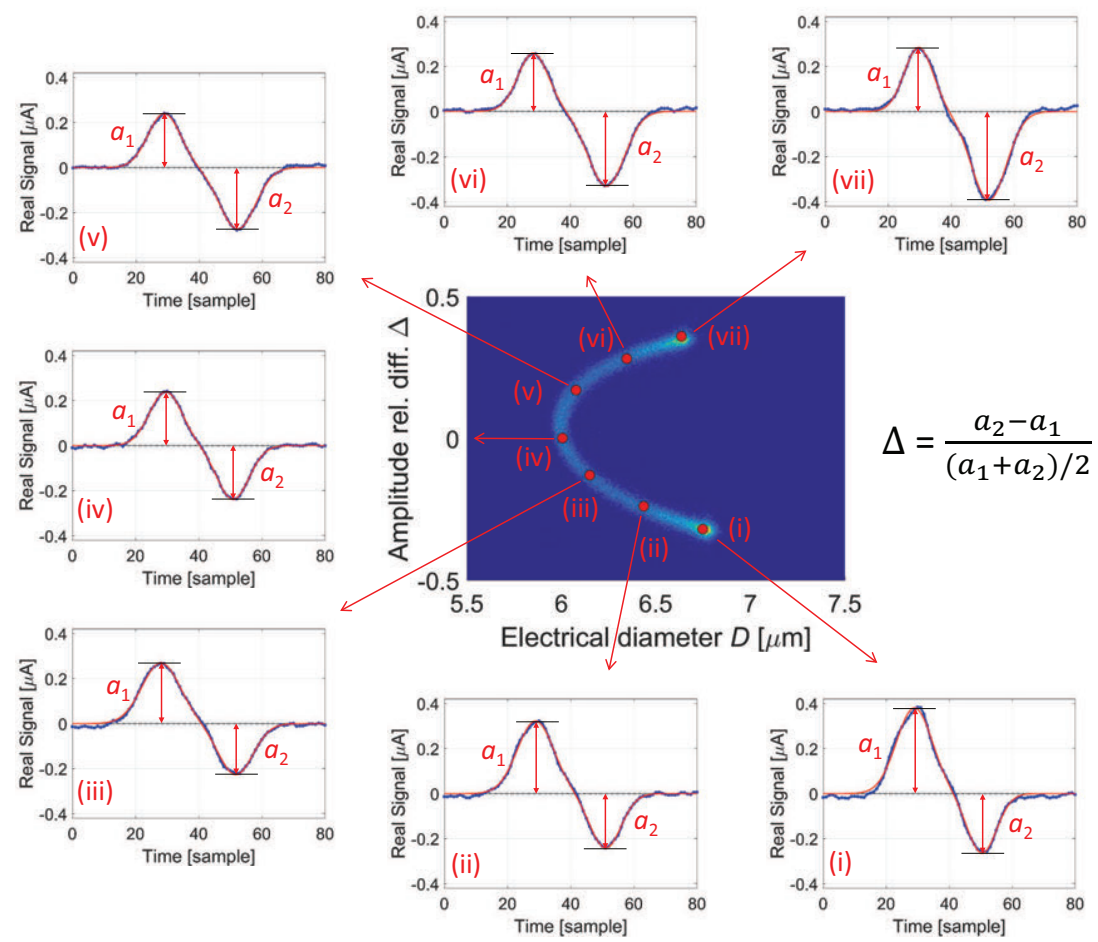

Fig. 3. Proposed wiring scheme. Density plot for $6 \mu \mathrm{m}$ diameter beads, with the pulse amplitude relative difference $\Delta$ plotted against the electrical diameter D. (i)-(vii) are experimental single particle signals (blue line) for the data points in the density plot. Fitting templates are also shown (red line).

where $a$ is particle nominal diameter, and the constants $b$ and $c$ account for the variation in signal with particle height as determined from the metric $\Delta$. These fitting parameters are listed in Table 1 , and the corresponding parabolas are shown in red in Fig. 4. The constant, $c$ should vanish for a channel ideally symmetric top to bottom. The fitted values are in fact very small (possibly due to minor channel asymmetry). The constants, $b$ and $c$ should be independent of particle sizes, which is clear from Table 1 where the differences are minor. The mean value for constants $b$ and $c$ was then used to calculate the parabolas shown in Fig. 4(d) for the three different particle sizes, showing an excellent fit with the data.

Fig. 5(b) and (c) shows histograms of the electrical diameter $D$ ob- 
(a)

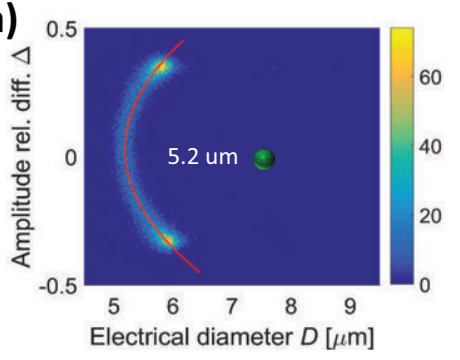

(b)

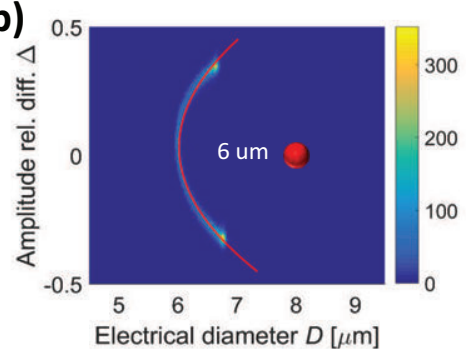

(c)

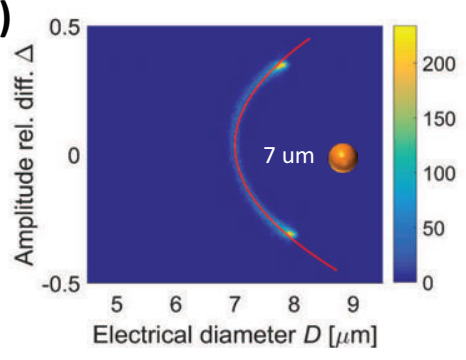

(d)

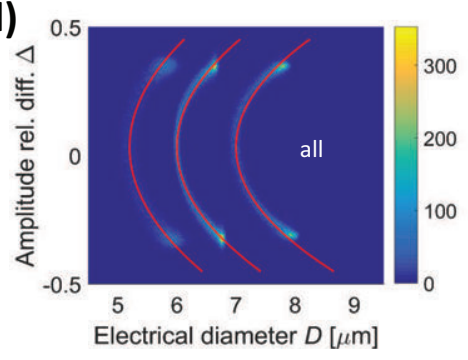

Fig. 4. Proposed wiring scheme. Density plot for populations of beads of different sizes, with the pulse amplitude relative difference $\Delta$ plotted against the electrical diameter $D$. (a) $5.2 \mu \mathrm{m}$ diameter beads, (b) $6 \mu \mathrm{m}$ diameter beads, (c) $7 \mu m$ diameter beads and (d) collection of the events in panels (a)-(c)plotted on the same graph. The fitted parabolas $D=a\left[1+b(\Delta-c)^{2}\right]$ are shown as red lines. For each population, parameter values $a, b$, and $c$ are reported in Table 1. In (d), the three parabolas are defined with $a=5.2,6.0,7.0 \mu \mathrm{m}$ and the same parameters $b$ and $c$ (average values, Table 1).

TABLE 1. Parameters of quadratic model equation $D=a[1+b(\Delta-$ $c)^{2}$ ] used to fit data plotted in Fig. 4 ( $95 \%$ confidence interval within the uncertainty in the least significant digit).

\begin{tabular}{llll}
\hline$d \quad[\mu \mathrm{m}]$ & $a[\mu \mathrm{m}]$ & $b$ & $c$ \\
\hline 5.2 & 5.19 & 1.06 & 0.028 \\
6.0 & 6.02 & 0.94 & 0.032 \\
7.0 & 7.00 & 1.04 & 0.035 \\
Mean & --- & 1.01 & 0.032 \\
\hline
\end{tabular}


(a)

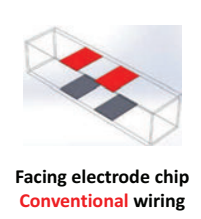

(d)

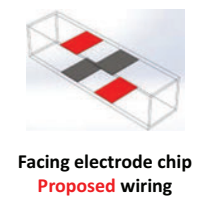

(g)

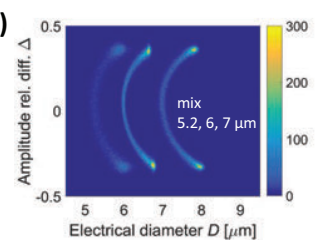

(b)

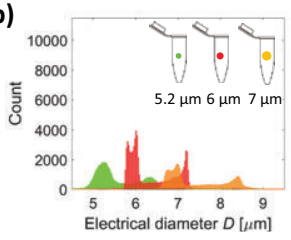

(c)

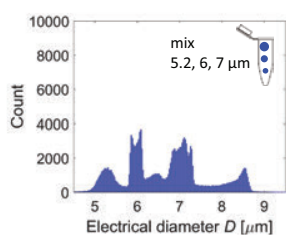

(e)

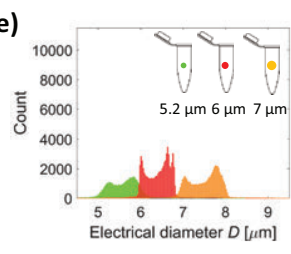

(h)

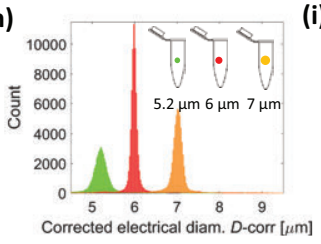

(f)

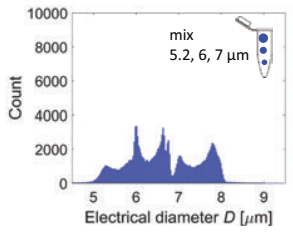

(i)

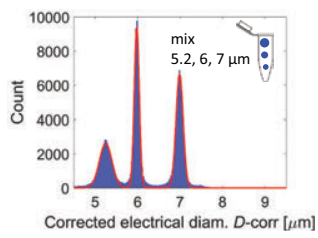

Fig. 5. (a)-(c) Conventional operation mode. (a) 3D view cartoon. Histogram of the electric diameter $D$ of 5.2, 6, and $7 \mu m$ diameter beads measured (b) separately and (c) together. (d)-(i) Proposed operation mode. (d) $3 D$ view cartoon. (g) Density plot of the pulse amplitude relative difference $\Delta$ plotted against the electrical diameter $D$ for a mixture of 5.2, 6, and $7 \mu m$ diameter beads. (e), (f) Histogram of the electric diameter $D$ of 5.2, 6, and $7 \mu m$ diameter beads measured (e) separately and (f) together. (b),(i) Histogram of the corrected electric diameter D-corr, showing an almost perfect Gaussian distribution.

tained using the conventional wiring scheme, relevant to individual particle populations and the mixed sample, respectively. As expected, the distribution has a significant spread and skewness, with overlap between the three populations.

Fig. 5(e) and (f) shows the analogous histograms obtained using the proposed wiring scheme. The spread and skewness are mitigated, because the cross contribution to the differential current was removed by averaging the pulse amplitudes according to equation (3). However, they are 
still present. Equation (5) was used to correct the raw data as follows:

$$
D \text {-corr }=\frac{D}{1+b(\Delta-c)^{2}},
$$

where $b$ and $c$ are the mean values of the constants in Table 1 . The corrected data is plotted in Fig. 5(h) and (i) showing an almost perfect Gaussian distribution. Fitting a Gaussian allows the coefficients of variation (CVs) to be calculated as follows: $2.82 \%, 0.98 \%$, and $1.20 \%$, for the 5.2 , 6 , and $7 \mu \mathrm{m}$ diameter beads respectively. This can be compared with the manufacturers' quoted values of $2.6 \%, 10 \%$, and $1.7 \%$.

Summarizing, the widely used microfluidic impedance chips comprising two pairs of electrodes are promoted to a superior level of accuracy by simply swapping two connection wires. With the novel wiring scheme, a left-right asymmetry is introduced in the system that is profitably exploited to compensate for positional dependence of the measured signals. This novel concept may open new opportunities for impedance cytometry in applications requiring accurate particle sizing and characterization, such as cell deformation measurement under applied load.

\section{Electrodeformation of RED BLOOD CELLS}

In order to identify the optimal design of an assay for single-cell mechanical characterization and to assist the interpretation of experimental results, an important role is played by modeling and simulation strategies. In particular, in case of electro-deformation systems, modeling and simulation can provide an accurate estimate of the mechanical forces induced on the cell by the electric field, and the consequent deformation on the basis of the cell mechanical behavior.

Within the cell mechanics literature, the erythrocyte or red blood cell (RBC) is probably the most studied cell type. As discussed in [12], this prominent role is ascribable to the simple structure of the erythrocyte, which is essentially a biconcave liquid capsule enclosed by a membrane, as well as to the importance of RBC deformability in pathophysiology. In fact, erythrocyte deformability is crucial to microvascular function and becomes altered e.g. in malaria, sickle cell anaemia, diabetes mellitus, and cardiovascular disease [23].

Besides other techniques (e.g., micropipette aspiration, optical tweezers experiments, deformation in shear or Poiseuille flows), cell deforma- 


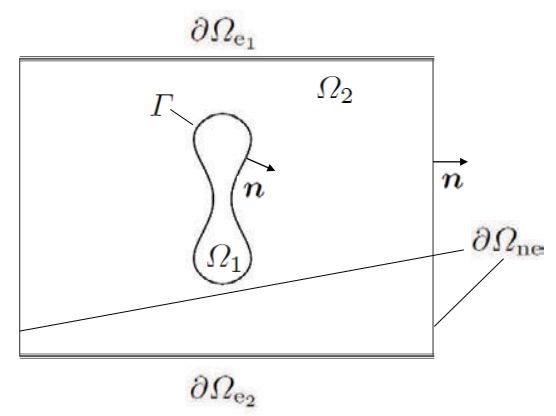

Fig. 6. Domain of the electric problem (schematic $2 D$ representation): $\Omega_{1}$, intracellular space; $\Omega_{2}$ suspending fluid; $\Gamma$, cell membrane; $\partial \Omega_{n e}$, boundary not covered by electrodes; $\partial \Omega_{e_{i}}, i$-th electrode; $\mathbf{n}$ normal unit vector

tion induced by electric fields is especially attractive due to its easiness of implementation. Here a computational approach to electro-deformation for erythrocyte mechanical characterization is presented. In particular, a finite-element based simulation tool is developed, composed of two coupled modules, namely, the electrical module and the mechanical one. The former is devoted to the computation of the mechanical actions induced by the electric field on the RBC (for a given geometry), whereas the latter computes the RBC deformation under a prescribed (electric) load. A fixed-point iteration scheme is adopted to implement the electromechanical coupling.

\subsection{Electrical module}

A RBC suspended in a fluid is here considered. In presence of an applied electric field, electric charges are induced on the cell membrane, and the interaction of the field with those charges gives rise to mechanical actions responsible for various frequency-dependent behaviours, such as orientation, translation (dielectrophoresis), rotation, and deformation. The purpose of the electrical module is the computation of these mechanical actions.

The investigation of field-induced actions has long been subject of research (see e.g. $[38,37]$ ) and different strategies have been proposed for their evaluation. Here the Maxwell stress tensor formulation is adopted, which is regarded in the literature as the most general and rigorous approach [39].

As pictured in Fig. 6, the electrical domain is modeled as the union 
of two homogeneous conducting regions $\Omega_{1}$ and $\Omega_{2}$, representing the intracellular space and the suspending fluid, respectively. Their complex conductivities $\sigma_{1}^{*}$ and $\sigma_{2}^{*}$ are given by $\sigma_{k}^{*}=\sigma_{k}+\mathrm{i} \omega \varepsilon_{k} \varepsilon_{\mathbf{V}}, k=1$, 2 , where $\varepsilon_{\mathrm{V}}$ is the vacuum permittivity, and $\sigma_{k}$ and $\varepsilon_{k}$ are the conductivity and relative permittivity of the media, respectively; moreover, $i$ is the imaginary unit, and $\omega$ denotes the circular frequency. The cell membrane, which is a thin phospholipid bilayer, is treated as a two-dimensional interface $\Gamma$ with conductance $G$ and capacitance $C$ per unit area, respectively given by the electric conductivity $\sigma_{\mathrm{m}}$ and permittivity $\varepsilon_{\mathrm{m}}$ of the lipid bilayer, divided by its thickness [1]. Therefore, the interface admittance per unit area is $Y=G+\mathrm{i} \omega C$. In the radio-frequency range $Y \approx \mathrm{i} \omega C$, since $G \ll \omega C[21]$, and hence the cell membrane essentially behaves like a capacitor. The finite interface admittance causes the electric potential to jump across the cell membrane [1]. The boundary of the domain is divided into an insulating part $\left(\partial \Omega_{\mathrm{ne}}\right)$, and a part covered by electrodes $\left(\partial \Omega_{\mathrm{e}}\right)$ which generate the electric field.

Field-induced actions can be derived by computing the variation of the electrostatic energy $\mathcal{E}_{\text {el }}$ resulting from a virtual variation of configuration [33], i.e.,

$$
\delta \mathcal{E}_{\text {el }}=\int_{\Omega_{1} \cup \Omega_{2}}-\mathbf{f} \cdot \delta \mathbf{s} \mathrm{d} V+\int_{\Gamma}-\mathbf{f}_{\Gamma} \cdot \delta \mathbf{s} \mathrm{d} A,
$$

where $\mathbf{f}$ and $\mathbf{f}_{\Gamma}$ denote volume and surface forces, respectively, and $\delta \mathbf{s}$ is a virtual displacement compactly supported in $\Omega_{1} \cup \Omega_{2}$. The variation $\delta \mathcal{E}_{\text {el }}$ is performed keeping constant the potential on the electrodes. Accordingly, the following energy functional is considered

$$
\mathcal{E}_{\text {el }}=\int_{\Omega_{1} \cup \Omega_{2}} \frac{1}{2} \varepsilon \mathbf{e} \cdot \mathbf{e} \mathrm{d} V+\int_{\Gamma} \frac{1}{2} C \llbracket \psi \rrbracket^{2} \mathrm{~d} A-\sum_{i} Q_{i} \psi_{i},
$$

where $\mathbf{e}$ is the electric field, $\psi$ is the electric potential, and $Q_{i}$ and $\psi_{i}$ denote the charge and the electric potential on the $i$-th electrode, respectively. Moreover, $\varepsilon=\varepsilon_{k} \varepsilon_{\mathrm{V}}$ in $\Omega_{k}, k=1,2$, the symbol $\cdot$ denotes the scalar product, and $\llbracket \cdot \rrbracket$ denotes the jump of the enclosed quantity across the membrane. The quasi-static approximation, $\mathbf{e}=-\nabla \psi$, is assumed, where $\nabla$ denotes the gradient operator. By resorting to the notion of con- 
figurational derivative [25], it can be shown that

$$
\delta \mathcal{E}_{\text {el }}=\int_{\Omega_{1} \cup \Omega_{2}} \mathbf{T}: \hat{\nabla} \delta \mathbf{s} \mathrm{d} V+\int_{\Gamma} t_{\Gamma}\left(\operatorname{div}_{\tau} \delta \mathbf{s}\right) \mathrm{d} A .
$$

Here $\hat{\nabla}$ and $\operatorname{div}_{\tau}$ denote the symmetric gradient and the tangential divergence operators, respectively, $\mathbf{T}$ is the Maxwell stress tensor

$$
\mathbf{T}=\varepsilon\left[\mathbf{e} \otimes \mathbf{e}-\frac{1}{2}|\mathbf{e}|^{2} \mathbf{I}\right],
$$

where $|\cdot|$ denotes the modulus, $\otimes$ is the tensor product, $\mathbf{I}$ is the identity tensor, and $t_{\Gamma}$ is given by

$$
t_{\Gamma}=-\frac{1}{2} C \llbracket \psi \rrbracket^{2} .
$$

The latter term, being dual to local area variations, can be regarded as a hydrostatic membrane stress, and does not induce any deformation due to the local area conservation hypothesis [28].

Upon integration by parts, the first term in (9) can be rewritten as

$$
\int_{\Omega_{1} \cup \Omega_{2}} \mathbf{T}: \hat{\nabla} \delta \mathbf{s} \mathrm{d} V=\int_{\Omega_{1} \cup \Omega_{2}}-\operatorname{div} \mathbf{T} \cdot \delta \mathbf{s} \mathrm{d} V+\int_{\Gamma}-\llbracket \mathbf{T n} \rrbracket \cdot \delta \mathbf{s} \mathrm{d} A,
$$

yielding the volume forces $\mathbf{f}$ and the surface forces $\mathbf{f}_{\Gamma}$ induced by the electric field as

$$
\mathbf{f}=\operatorname{div} \mathbf{T}, \quad \mathbf{f}_{\Gamma}=\llbracket \mathbf{T} \mathbf{n} \rrbracket,
$$

where div denotes the divergence operator and $\mathbf{n}$ is the normal unit vector to $\Gamma$ pointing into $\Omega_{2}$. It is a simple matter to show that [33]

$$
\mathbf{f}=-\frac{1}{2}(\nabla \varepsilon) \mathbf{e} \cdot \mathbf{e} .
$$

As a consequence, under the assumption of constant permittivities, volume forces vanish and only the surface forces $f_{\Gamma}$ acting on the cell membrane have to be considered as load in the mechanical module. From $(13)_{2}$ and (10), their computation requires the knowledge of the electric field distribution. Moreover, in case of time-harmonic electric field

$$
\mathbf{e}=\mathbf{A} \cos (\omega t+\varphi),
$$


(a)

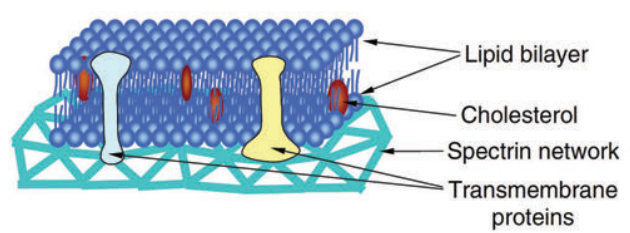

(b)

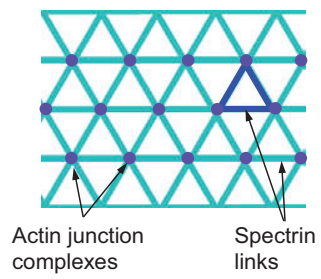

(c)

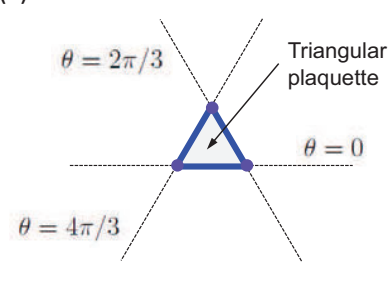

Fig. 7. (a) Schematic drawing of the RBC membrane structure (not to scale).

(b) Idealised regular triangular network. (c) Representative triangle

the time-averaged counterpart of the Maxwell stress tensor has to be adopted [39,37], given by

$$
<\mathbf{T}>=\frac{1}{4} \varepsilon\left[(\mathbf{E} \otimes \overline{\mathbf{E}}+\overline{\mathbf{E}} \otimes \mathbf{E})-|\mathbf{E}|^{2} \mathbf{I}\right],
$$

where the overline denotes complex conjugation and $\mathbf{E}=\mathbf{A} \mathrm{e}^{\mathrm{i} \varphi}$ is the electric field phasor, which can be computed as detailed in [30].

\subsection{Mechanical module}

The structure of a RBC can be characterized as a liquid capsule enclosed by a biologic membrane. This membrane is composed of a phospholipid bilayer and an underlying spectrin network, the cytoskeleton, coupled to each other by integral membrane proteins (Fig. 7 ).

From a mechanical point of view, the spectrin network accounts for the in-plane shear stiffness of the membrane. On the other hand, the lipid bilayer resembles a fluid-like membrane, and is responsible of membrane bending stiffness and total surface area conservation [28]. Moreover, assuming that the connection between spectrin network and lipid bilayer is dense enough, the stricter constraint of local surface area conservation can be enforced [20]. Finally, the nearly incompressible cytosol inside the 
RBC determines the total volume conservation constraint [15].

Such arguments motivate the definition of the following energy potential, which governs the quasi-static mechanical evolution of the RBC $[15,20]$ :

$$
\mathcal{E}=W_{\text {in-plane }}+W_{\text {bend }}+W_{\text {vol }}+\mathcal{E}_{\text {ext }},
$$

where $W_{\text {in-plane }}$ and $W_{\text {bend }}$ denote the membrane in-plane and bending energies, respectively, $W_{\text {vol }}$ is a volume penalty energy term imposing the total volume conservation constraint and $\mathcal{E}_{\text {ext }}$ is the potential of external loads. The local surface area conservation constraint is included in the potential (17) as a contribution to the in-plane energy $W_{\text {in-plane }}$.

Introducing the in-plane energy density $w_{\text {in-plane }}$ per unit reference area $[14,2]$ and the bending energy density $w_{\text {bend }}$ per unit current area [24], it follows that:

$$
W_{\text {in-plane }}=\int_{A_{0}} w_{\text {in-plane }} \mathrm{d} A, \quad W_{\text {bend }}=\int_{A} w_{\text {bend }} \mathrm{d} A,
$$

where $A_{0}$ [resp. $\left.A\right]$ is the surface area in the reference [resp. current] configuration. The volume penalty energy term is defined as [15]:

$$
W_{\mathrm{vol}}=\frac{1}{2} k_{\mathrm{vol}} \frac{\left(V-V_{0}\right)^{2}}{V_{0}},
$$

where $V_{0}$ [resp. $V$ ] is the total cell volume in the reference [resp. current] configuration and $k_{\mathrm{vol}}$ is a suitable penalty parameter.

The definition of the RBC membrane constitutive law consists in the selection of in-plane and bending energy densities. In particular, starting from the so-called spectrin network model $[14,2]$, the in-plane energy density $w_{\text {in-plane }}$ is obtained by means of homogenization of the Helmholtz free energy of the microstructure [30]. Moreover, the bending energy density $w_{\text {bend }}$ is assumed to obey the Helfrich model [24].

The mechanical problem is solved using the finite element implementation proposed in [30], adopting a rotation-free shell element, whose development profitably exploits a corotational formulation [3,4]. An alternative solution, based on isogeometric analysis, can be found in [31].

\subsection{Electro-mechanical coupling}

The coupling between the electrical and mechanical modules is implemented by means of a fixed-point iteration scheme (Fig. 8). For given ap- 


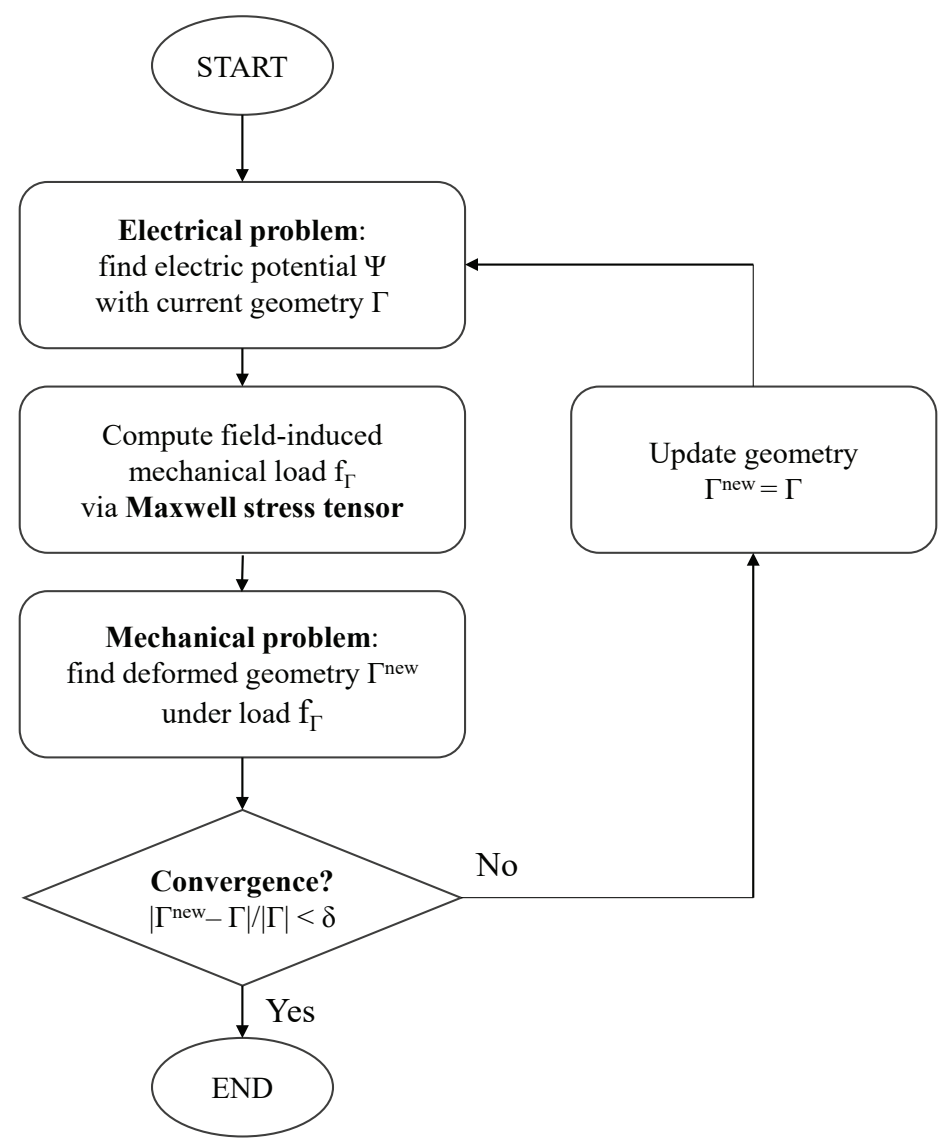

Fig. 8. Flow chart of electro-mechanical coupling (see text for explanation)

plied electrode potentials $\Psi_{i}$, the electric problem is initially solved on the domain defined by the reference configuration of the cell membrane $\Gamma$. The corresponding field-induced actions $\mathbf{f}_{\Gamma}$ are then computed as the jump of the tractions generated by the time-averaged Maxwell stress tensor on the cell membrane (equations $(13)_{2}$ and (10)). The latter surface forces define the potential of the external load $\mathcal{E}_{\text {ext }}$ in the mechanical problem (17), whose solution provides an updated cell-membrane geometry $\Gamma^{\text {new }}$. If the distance between $\Gamma$ and $\Gamma^{\text {new }}$ is lower than a prescribed relative tolerance $\delta$, the numerical procedure stops, otherwise, a new it- 
(a)

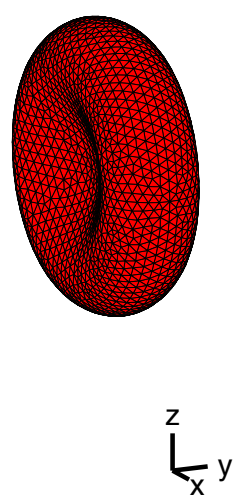

(b)

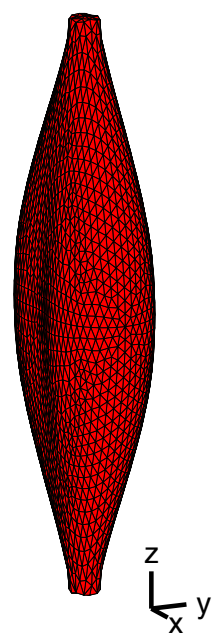

Fig. 9. Numerical simulation of the large deformation of a RBC using optical tweezers. (a) Reference configuration. (b) Deformed configuration under maximum load $\left(F_{\max }=200 p N\right)$

eration is performed starting from the updated cell-membrane geometry $\Gamma^{\text {new }}$. In particular, in the convergence check, the norm of the relative difference between the nodal coordinates of $\Gamma$ and $\Gamma^{\text {new }}$ is considered, with a relative tolerance $\delta=0.001$.

\subsection{Numerical results}

\subsubsection{Large deformation of the $R B C$ by optical tweezers}

Deformation imposed by optical tweezers provides a useful means for the study of single cell mechanics under a variety of well-controlled stressstates [29]. While early studies involved primarily small elastic deformation at low applied forces, the possibility of inducing large elastic deformation in human RBCs using optical tweezers was demonstrated in $[12,29]$. In those papers, forces as high as about $193 \pm 20 \mathrm{pN}$ were estimated to result in strains of the order of $100 \%$ in the cell. The experimental procedure adopted to stretch the cell is as follows. Two silica microbeads are non-specifically attached to the RBC at diametrically opposite points. One bead is anchored to the surface of a glass slide, the other 


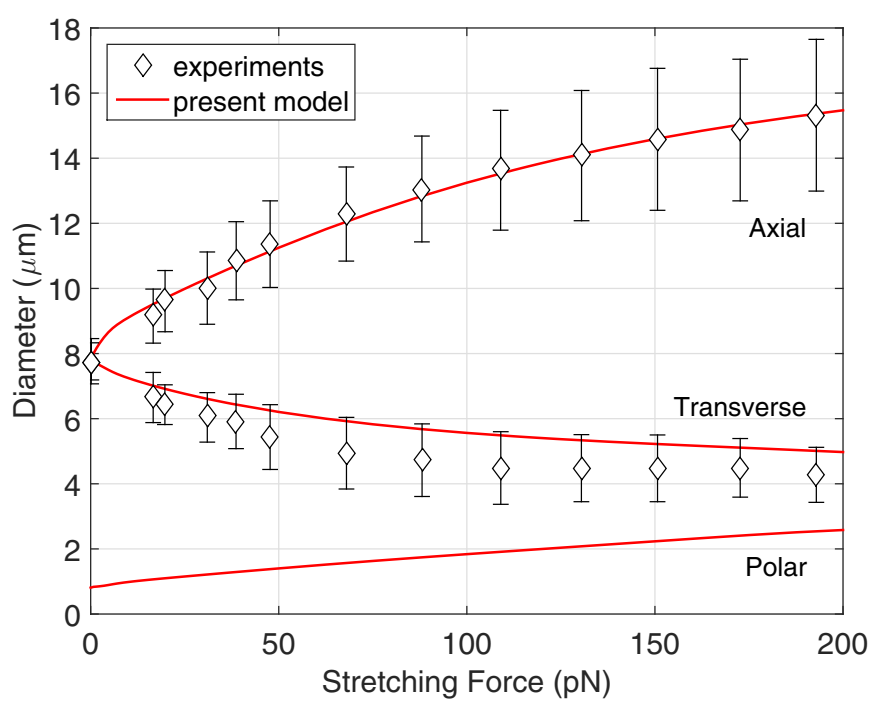

Fig. 10. Diameters versus stretching force during the large deformation of a $R B C$ using optical tweezers. The present numerical results are compared with the experimental data reported in [29] (not including the polar diameter)

TABLE 2. Parameters of the RBC meridional section [19]

\begin{tabular}{llll}
\hline$R(\mu \mathrm{m})$ & $C_{0}$ & $C_{1}$ & $C_{2}$ \\
\hline 3.91 & 0.207161 & 2.002558 & -1.122762 \\
\hline
\end{tabular}

one is trapped using a laser beam. While the trapped bead remains stationary, moving the slide with the anchored bead stretches the cell.

In the in-silico experiment, the biconcave RBC geometric model (Fig. 9(a)) was obtained by revolution of the meridional section [19]:

$$
f(r)=\frac{R}{2} \sqrt{1-\left(\frac{r}{R}\right)^{2}}\left[C_{0}+C_{1}\left(\frac{r}{R}\right)^{2}+C_{2}\left(\frac{r}{R}\right)^{4}\right],
$$

whose parameter values are reported in Table 2. Parameters characterizing the $\mathrm{RBC}$ mechanical behaviour adopted in the simulations can be found in [30]. The load imposed by each silica bead was simulated as a force $F$ distributed on a circular contact area of diameter $D=1.1 \mu \mathrm{m}$. Equal $z$-displacement was imposed at the interested nodes. Sixteen uni- 


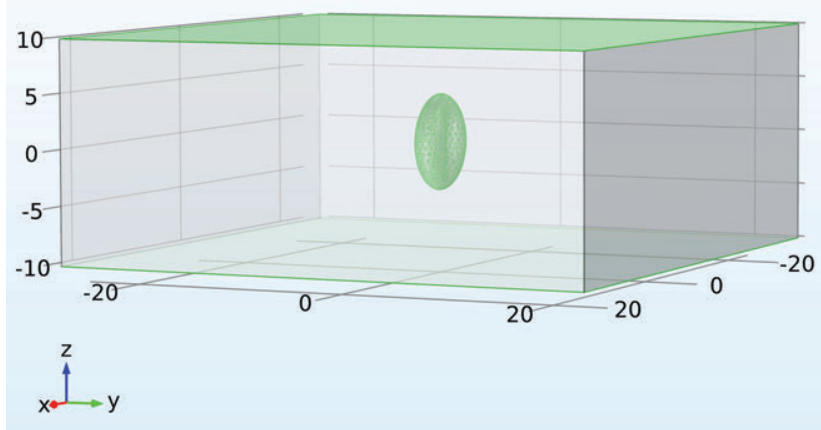

Fig. 11. In silico electro-deformation experiment: a RBC, suspended in a conducting medium, is placed between two facing electrodes, and a voltage is applied between them. (COMSOL geometric model)

form load-steps, with internal adaptive sub-stepping, were considered, up to a maximum force level $F_{\max }=200 \mathrm{pN}$. Due to symmetry, one-eight of the cell was studied, with a mesh of 524 elements.

Fig. 9(b) shows the deformed configuration of the RBC under maximum load. The behaviour of transverse, axial and polar diameters as functions of the applied force is shown in Fig. 10, where the experimental data provided in [29] are also reported for comparison. Excellent agreement is found for the axial diameter, whereas a small discrepancy is noticed between simulated and experimental transverse diameters, with experimental data providing lower values. As suggested in [20], this is probably a consequence of the optical measurements being performed from only a single observation angle, that may result in under-prediction of the maximum transverse diameter in presence of rotation of the $\mathrm{RBC}$ during the stretch test. However, the simulation results remain within the experimental error bars. The behaviour of the polar diameter, not reported in the experimental data, highlights a progressive change from the cell biconcave shape to a more rounded shape. Generally, a hardening behaviour with increasing force is noticed, mainly due to the nonlinear constitutive properties of the spectrin network. 
(a)

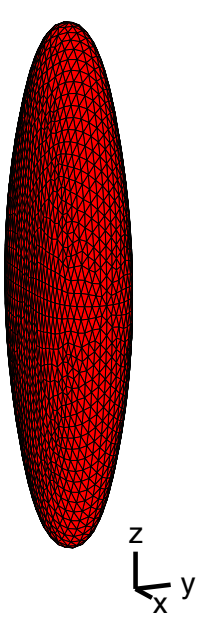

(b)

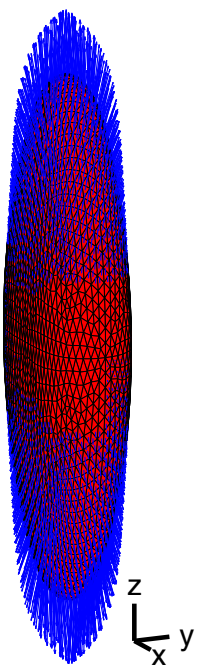

Fig. 12. Electro-deformation of a RBC. (a) Deformed configuration under maximum applied potential ( $\Psi_{\max }=2.5 \mathrm{~V} @ 500 \mathrm{kHz}$ ). (b) Visualization of field-induced mechanical actions

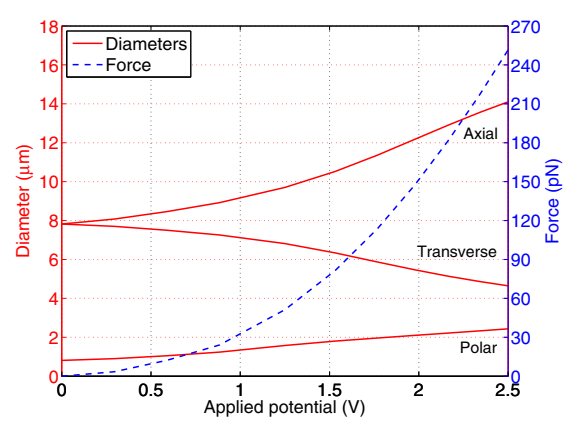

Fig. 13. Diameters and half-cell resultant force versus applied potential during the electro-deformation of a $R B C$

\subsubsection{Electro-deformation of a $R B C$}

A RBC, suspended in a conducting medium, was placed between two $50 \mu \mathrm{m} \times 50 \mu \mathrm{m}$ facing electrodes, that are $20 \mu \mathrm{m}$ apart from each other (Fig. 11). A voltage $\Psi$ at $500 \mathrm{kHz}$ was applied between the electrodes. As typical in electro-deformation experiments, a poorly conductive extracel- 
lular fluid was considered [17]. Due to symmetry, one-eight of the system was studied.

In order to obtain an efficient implementation of the electromechanical coupling, the same mesh was adopted to discretize the cell surface in the electrical and mechanical modules. It was composed by 524 triangular elements. Starting from this mesh, a mesh composed by 397,851 quadratic tetrahedral elements was generated to discretize the electric domain. Eight applied-potential steps were considered, with quadratic spacing, up to a maximum value $\Psi_{\max }=2.5 \mathrm{~V}$.

The average number of fixed-point iterations in each load step, needed to reach convergence in the electro-mechanical coupling scheme, was 3.4.

Fig. 12(a) shows the deformed configuration under maximum applied potential, and the mechanical actions induced by the electric field are visualized in Fig. 12(b). The magnitude of the resultant force on the top and bottom half cells is shown in Fig. 13 as function of the applied voltage $\Psi$. The same figure also reports the axial, transverse, and polar diameters of the deformed RBC. Under the maximum applied voltage, a force magnitude of $252 \mathrm{pN}$ was computed, yielding a deformed RBC configuration with diameters of $14.1 \mu \mathrm{m}, 4.64 \mu \mathrm{m}$, and $2.44 \mu \mathrm{m}$, respectively.

Electro-deformation has been recently employed in conjunction with microfluidic systems for biomechanical measurements of RBCs and other cell types $[16,17,27]$. An estimate of the applied resultant force on half cell is usually computed and related to the experimentally-measured deformation, whence phenomenological indicators of the cell mechanical behaviour are derived. In this context, the modeling and simulation framework here developed represents a valuable tool. In fact, it provides an accurate estimate of the distribution of field-induced actions for cells with arbitrary geometry and without simplifying assumptions on the electric field distribution. This feature can be of aid in the design of optimal electro-deformation systems (e.g. in terms of geometry, medium properties and frequency).

\section{Conclusions}

Experimental and computational mechanics provide a detailed understanding about essential connections among structures, mechanical prop- 
erties and functions of cells. Although many efforts in cell mechanics have already been aimed towards understanding how cells move, sense, deform and interact with their microenvironment, there is a pressing need to study how the mechanical properties of cells change during a state of disease and how these changes impact signaling processes [36]. Emerging microscale technologies are opening new venues in studying mechanical aspects of cells due to their cost-effectiveness, relatively easy fabrication, and high throughput. The incorporation of mechanical, electrical, optical and information technologies has great potential for developing efficient, versatile and comprehensive analysis tools at the single-cell level.

\section{REFERENCES}

[1] Bisegna, P., Caselli, F.: A simple formula for the effective complex conductivity of periodic fibrous composites with interfacial impedance and applications to biological tissues. J Phys D: Appl Phys 41, 115,506 (2008). DOI 10.1088/00223727/41/11/115506

[2] Boey, S.K., Boal, D.H., Discher, D.E.: Simulations of the erythrocyte cytoskeleton at large deformation. I. Microscopic models. Biophys J 75(3), 1573-1583 (1998). DOI 10.1016/S0006-3495(98)74075-5

[3] Caselli, F., Bisegna, P.: Polar decomposition based corotational framework for triangular shell elements with distributed loads. Int J Numer Methods Eng 95(6), 499-528 (2013). DOI 10.1002/nme.4528

[4] Caselli, F., Bisegna, P.: A corotational flat triangular element for large strain analysis of thin shells with application to soft biological tissues. Comput Mech 54(3), 847-864 (2014). DOI 10.1007/s00466-014-1038-9

[5] Caselli, F., Bisegna, P.: A simple and robust event- detection algorithm for singlecell impedance cytometry. IEEE Trans Biomed Eng 63(2), 415-422 (2016). DOI 10.1109/TBME.2015.2462292

[6] Caselli, F., Bisegna, P., Maceri, F.: EIT-inspired microfluidic cytometer for singlecell dielectric spectroscopy. J Microelectromech Syst 19(5), 1029-1040 (2010). DOI 10.1109/JMEMS.2010.2067204

[7] Caselli, F., Ninno, A.D., Reale, R., Businaro, L., Bisegna, P.: A novel wiring scheme for standard chips enabling high-accuracy impedance cytometry. Sensors and Actuators B: Chemical 256, 580 - 589 (2018). DOI 10.1016/j.snb.2017.10.113

[8] Caselli, F., Reale, R., Nodargi, N.A., Bisegna, P.: Numerical investigation of a novel wiring scheme enabling simple and accurate impedance cytometry. Micromachines 8(9), 283 (2017). DOI 10.3390/mi8090283

[9] Caselli, F., Shaker, M., Colella, L., Renaud, P., Bisegna, P.: Modeling, simulation, and performance evaluation of a novel microfluidic impedance cytometer for morphology-based cell discrimination. J Microelectromech Syst 23(4), 785-794 
(2014). DOI 10.1109/JMEMS.2014.2325979

[10] Cheung, K.C., Di Berardino, M., Schade-Kampmann, G., Hebeisen, M., Pierzchalski, A., Bocsi, J., Mittag, A., TÃ,Â'arnok, A.: Microfluidic impedance-based flow cytometry. Cytometry Part A 77(7), 648-66 (2010)

[11] Cross, S.E., Jin, Y.S., Tondre, J., Wong, R., Rao, J., Gimzewski, J.K.: AFM-based analysis of human metastatic cancer cells. Nanotechnology 19(38), 384,003

[12] Dao, M., Lim, C.T., Suresh, S.: Mechanics of the buman red blood cell deformed by optical tweezers. J Mech Phys Solids 51(11-12), 2259-2280 (2003). DOI 10.1016/j.jmps.2003.09.019

[13] Di Carlo, D.: A mechanical biomarker of cell state in medicine. J Lab Autom 17(1), 32-42 (2012). DOI 10.1177/2211068211431630

[14] Discher, D.E., Boal, D.H., Boey, S.K.: Phase transitions and anisotropic responses of planar triangular nets under large deformation. Phys Rev E 55(4), $4762-4772$ (1997). DOI 10.1103/PhysRevE.55.4762

[15] Discher, D.E., Boal, D.H., Boey, S.K.: Simulations of the erythrocyte cytoskeleton at large deformation. II. Micropipette aspiration. Biophys J 75(3), 1584-1597 (1998). DOI 10.1016/S0006-3495(98)74076-7

[16] Doh, I., Lee, W.C., Cho, Y.H., Pisano, A.P., Kuypers, F.A.: Deformation measurement of individual cells in large populations using a single-cell microchamber array chip. Appl Phys Lett 100(17) (2012). DOI 10.1063/1.4704923

[17] Du, E., Dao, M., Suresh, S.: Quantitative biomechanics of healthy and diseased buman red blood cells using dielectrophoresis in a microfluidic system. Extr Mech Lett 1, 35-41 (2014). DOI 10.1016/j.eml.2014.11.006

[18] Errico, V., De Ninno, A., Bertani, F., Businaro, L., Bisegna, P., Caselli, F.: Mitigating positional dependence in coplanar electrode coulter-type microfluidic devices. Sens. Act. B Chem. 247, 580-586 (2017). DOI 10.1016/j.snb.2017.03.035

[19] Evans, E., Fung, Y.C.: Improved measurements of erythrocyte geometry. Microvasc Res 4(4), 335-347 (1972). DOI 10.1016/0026-2862(72)90069-6

[20] Fedosov, D.A., Caswell, B., Karniadakis, G.E.: A multiscale red blood cell model with accurate mechanics, rheology, and dynamics. Biophys J 98(10), 2215-2225 (2010). DOI 10.1016/j.bpj.2010.02.002

[21] Foster, K.R., Schwan, H.P.: Dielectric properties of tissues and biological materials: a critical review. Crit Rev Biomed Eng 17(2), 25-104 (1989)

[22] Gawad, S., Cheung, K., Seger, U., Bertsch, A., Renaud, P.: Dielectric spectroscopy in a micromachined flow cytometer: theoretical and practical considerations. Lab Chip 4, 241-251 (2004). DOI 10.1039/b313761a

[23] Haque, M.M.: Elastic theory for the deformation of a spherical dielectric biological object under electro-optical trapping. RSC Adv 5(55), 44,458-44,462 (2015). DOI 10.1039/C5RA06125C

[24] Helfrich, W.: Elastic properties of lipid bilayers: theory and possible experiments. Z Naturforsch C 28(11), 693-703 (1973)

[25] Kirsch, A.: The domain derivative and two applications in inverse scattering theory. Inverse Prob 9(1), 81-96 (1999). DOI 10.1088/0266-5611/9/1/005 
[26] LaFratta, C.N.: Optical tweezers for medical diagnostics. Analytical and Bioanalytical Chemistry 405(17), 5671- 5677 (2013). DOI 10.1007/s00216-013-6919-9

[27] Leung, S.L., Lu, Y., Bluestein, D., Slepian, M.J.: Dielectrophoresis-mediated electrodeformation as a means of determining individual platelet sti $\hat{A}^{-} \hat{A} \neg \hat{A} \pm n e s s$. Ann Biomed Eng pp. 1-11 (2015). DOI 10.1007/s10439-015-1383-7

[28] Li, J., Dao, M., Lim, C.T., Suresh, S.: Spectrin-level modeling of the cytoskeleton and optical tweezers stretching of the erythrocyte. Biophys J 88(5), 3707-3719 (2005). DOI 10.1529/biophysj.104.047332

[29] Mills, J.P., Qie, L., Dao, M., Lim, C.T., Suresh, S.: Nonlinear elastic and viscoelastic deformation of the human red blood cell with optical tweezers. Mech Chem Biosyst 1(3), 169-180 (2004)

[30] Nodargi, N.A., Bisegna, P., Caselli, F.: E $\tilde{A}-\hat{A} \neg \hat{A}$ Eective computational modeling of erythrocyte electro-deformation. Meccanica 52(3), 613-631 (2017). DOI 10.1007/s11012- 016-0424-0

[31] Nodargi, N.A., Kiendl, J., Bisegna, P., Caselli, F., Lorenzis, L.D.: An isogeometric analysis formulation for red blood cell electro-deformation modeling. Computer Methods in Applied Mechanics and Engineering 338, 392-411 (2018). DOI 10.1016/j.cma.2018.04.038

[32] Spencer, D., Morgan, H.: Positional dependence of particles in microfludic impedance cytometry. Lab Chip 11(7), 1234-39 (2011)

[33] Stratton, J.A.: Electromagnetic Theory. McGraw-Hill (1941)

[34] Sun, T., Green, N.G., Gawad, S., Morgan, H.: Analytical electric field and sensitivity analysis for two microfluidic impedance cytometer designs. IET Nanobiotechnol. 1(5), 69-79 (2007). DOI 10.1049/iet-nbt:20070019

[35] Sun, T., Morgan, H.: Single-cell microfluidic impedance cytometry: a review. Microfluid Nanofluid 8(4), 423-43 (2010)

[36] Unal, M., Alapan, Y., Jia, H., Varga, A.G., Angelino, K., Aslan, M., Sayin, I., Han, C., Jiang, Y., Zhang, Z., Gurkan, U.A.: Micro and nano-scale technologies for cell mechanics. Nanobiomedicine 1, 5 (2014). DOI 10.5772/59379

[37] Vlahovska, M.P., Gracià, R.S., Aranda-Espinoza, S., Dimova, R.: Electrobydrodynamic model of vesicle deformation in alternating electric fields. Biophys J 96(12), 4789-4803 (2009). DOI 10.1016/j.bpj.2009.03.054

[38] Voldman, J.: Electrical forces for microscale cell manipulation. Annu Rev Biomed Eng 8(1), 425-454 (2006). DOI 10.1146/annurev.bioeng.8.061505.095739

[39] Wang, X., Wang, X.B., Gascoyne, P.R.C.: General expressions for dielectrophoretic force and electrorotational torque derived using the Maxwell stress tensor method. J Electrostat 39(4), 277-295 (1997). DOI 10.1016/S0304-3886(97)00126-5

[40] Zhang, G., Long, M., Wu, Z., Yu, W.: Mechanical properties of hepatocellular carcinoma cells. World Journal Gastroenterol. 8(2), 243-246 (2002). DOI 10.3748/wjg.v8.i2.243 